\title{
Cerebral microembolism during atrial fibrillation ablation can result from the technical aspects and mostly does not cause permanent neurological deficit
}

\author{
Anetta Lasek-Bal'1, Przemysław Puz'1 Joanna Wieczorek², Seweryn Nowak², \\ Anna Maria Wnuk-Wojnar ${ }^{2}$, Aldona Warsz-Wianecka', Katarzyna Mizia-Stec ${ }^{2}$
}

\author{
'Department of Neurology, School of Health Sciences, Medical University of Silesia, \\ Katowice, Poland \\ ${ }^{2}$ First Department of Cardiology, School of Medicine, Medical University of Silesia, \\ Katowice, Poland
}

Submitted: 18 December 2019

Accepted: 7 March 2020

Arch Med Sci 2020; 16 (6): 1288-1294

DOI: https://doi.org/10.5114/aoms.2020.94747

Copyright $\odot 2020$ Termedia \& Banach

\begin{abstract}
Introduction: Atrial fibrillation ablation can be associated with microembolism detected in the intracranial arteries and risk of neurological incidents. The aims of this study were to evaluate microembolic signals (MES) during pulmonary vein isolation (PVI) and establish the potential significance of MES for damage of the brain in radiological investigation and neurological state. Material and methods: In the prospective study we included patients with atrial fibrillation undergoing percutaneous pulmonary vein isolation (radiofrequency ablation/balloon cryoablation) with ultrasound monitoring of $\mathrm{mi}$ croembolisms in the middle cerebral artery. Neurological examination and MRI of the head were performed in all participants.

Results: The study enrolled 80 patients at a mean age of 58 years. Microembolisms during the monitoring of the flow in the right middle cerebral artery were recorded in 61 (76.3\%) patients in the amount of 51-489 (mean: 239). Most often the microembolic signals were registered during the trans-septal puncture and the stage of ablation. In $89 \%$, microembolisms were gaseous. Mean score on the Fazekas scale for the whole group before ablation: 0.87 \pm 0.7 (0-3, med. 1); after: $0.93 \pm 0.71$. In 3 (4.3\%) patients the lesions worsened during the follow-up period. None of the patients revealed a cardiovascular event during the follow-up period and no changes were observed in the neurological status.

Conclusions: The majority of cerebral microembolisms generated during PVI are gaseous in nature. The cerebral microembolisms associated with PVI probably result from the technical aspects of the procedure and do not cause either permanent brain damage in the radiological investigation or neurological deficit.
\end{abstract}

Key words: ablation, pulmonary vein isolation, microembolism, microembolic signals, atrial fibrillation.

\section{Introduction}

Atrial fibrillation ablation is a recognized invasive method of treatment involving pulmonary vein isolation (PVI). The procedure performed within the left atrium using the insertion of electrodes by trans-septal puncture can be associated with the risk of acute neurological incidents

\author{
Corresponding author: \\ Anetta Lasek-Bal MD, PhD, \\ Prof. SUM \\ Department of Neurology \\ School of Health Sciences \\ Medical University \\ of Silesia \\ 45/47 Ziołowa St \\ 40-735 Katowice, Poland \\ Phone: +48 323598306 \\ Fax: +48 322029592 \\ E-mail: abal@sum.edu.pl
}


despite anticoagulation [1-3]. In the majority of patients, ultrasound monitoring of the blood flow in the middle cerebral arteries during PVI exhibits microembolic signals (MES) which are usually clinically asymptomatic both during detection and over short-term follow-up. However, $5-40 \%$ of patients in the periprocedural period reported acute vascular lesions in diffusion-weighted magnetic resonance imaging (DW-MRI, DWI) of the head [4-12]. Lesion morphology indicates an embolic, probably cardiogenic etiology. The presence of foci in DWI proves that MES can be manifested by means of radiology. To date, no studies have been carried out to evaluate the further evolution of MES. Although the significance of MES or foci in head DWI has not been established in the periprocedural period, some authors indicate a possible negative effect of MES during ablation on patients' cognitive functions [3, 10]. Evaluation of the procedure's safety is significant due to increasing numbers of ablations performed also in patients with multiple morbidities. Differences were demonstrated in the amount of recorded MES depending on the type of ablation; MES were significantly more frequent during standard radiofrequency (RF) PVI than during cryoballoon ablation $[5,6,13]$. It is conceivable that different types of atrial fibrillation ablation are associated with a different risk of acute neurological incidents $[1,3,5,6,12-18]$.

The aims of this study were to perform quantitative and a qualitative evaluation of MES during PVI and establish the potential significance of MES for damage of the brain assessed in radiological investigation and the neurological state of patients. Using the magnetic resonance technique, lesions of vascular origin were evaluated in patients before and up to a few months following the procedure. Ultrasound and radiological analyses were performed along with monitoring patients' neurological status.

\section{Material and methods}

The project was conducted as a prospective analysis. Patients with AF undergoing percutaneous PVI (radiofrequency ablation/balloon cryoablation) during scheduled hospitalization in the First Department of Cardiology, Medical University of Silesia were enrolled in the study group. Patients with paroxysmal and persistent $A F$, with no history of brain stroke or transient ischemic attack (TIA), were also included. The medical information was based on the tests and specialist consultations performed during hospitalization and/or the analysis of patients' previous medical records. Other eligibility criteria were: no prior ablation due to atrial fibrillation (AF), left ventricular ejection fraction (LVEF) > 50\%, no significant hemo- dynamic stenosis of the carotid ( $\geq 70 \%$ ) and/or cerebral artery ( $\geq 50 \%$ ) based on the result of duplex ultrasound with Doppler imaging. The exclusion criteria were: lack of adequate anticoagulation in the month preceding the procedure, prior ischemic or hemorrhagic stroke, venous or arterial thromboembolism, an estimated glomerular filtration rate $<60 \mathrm{ml} / \mathrm{min} / 1.73 \mathrm{~m}^{2}$, pregnancy and age $<18$ and $>80$. We used the $\mathrm{CHA}_{2} \mathrm{DS}_{2}$ VASc scale to estimate the annual burden of stroke. All patients were anticoagulated with vitamin $\mathrm{K}$ antagonists for at least one month prior to PVI, confirming once a week adequate anticoagulation in the month preceding the procedure. All patients underwent transesophageal echocardiography (TEE) in order to exclude the presence of LA appendage thrombus within $24 \mathrm{~h}$ prior to the procedure. Three or four venous accesses were obtained using the Seldinger technique - one for the left (6 Fr sheath - quadripolar electrode placed in the right ventricle) and right femoral vein (two $8 \mathrm{Fr}$ sheathes - circular mapping and radiofrequency ablation electrodes or one $14 \mathrm{Fr}$ for cryoablation balloon) and the right jugular vein (6 Fr sheath - decapolar electrode coronary sinus catheterization) (stage 1). Rotational angiography of the left atrium was performed at the beginning of the procedure (contrast medium injection into the pulmonary artery during rapid right ventricular stimulation). Trans-septal puncture was the next step of the procedure (stage 2). Following trans-septal puncture, 3-dimensional electro-anatomical mapping was performed in patients undergoing radiofrequency ablation using the CARTO 3 system (Biosense Webster, Diamond Bar, CA, USA). PVI was achieved by means of either $(n=72,90 \%)$ radiofrequency (RF) ablation with the ThermoCool SmartTouch SF catheter (Biosense Webster, Diamond Bar, CA, USA) or balloon cryoablation $(n=8$, $10 \%)$ using an Arctic Front Advance catheter (Medtronic, MN, USA) (stage 3).

Radiofrequency PVI ablation was performed with $30 \mathrm{~W}$ energy (reduction to $25 \mathrm{~W}$ in the posterior wall). The energy application in one spot was about $30 \mathrm{~s}$ with dragging to another for about 3$4 \mathrm{~mm}$ for the next $30 \mathrm{~s}$ circumferentially around all pulmonary veins. Isolation was defined as an entrance/exit block observed in a circular mapping electrode in every pulmonary vein. No steam pop was observed in patients treated using the workflow.

The final stage was the removal of devices outside the circulatory system (stage 4). PVI was performed under the guidance of a circular mapping electrode - Lasso (Biosense Webster, Diamond Bar, CA, USA) or Achieve catheter (Medtronic, MN, USA). The procedure was performed with an INR of up to 2.5 (preferably 2.0-2.5). 
All patients received an IV bolus of unfractionated heparin $(100 \mathrm{lU} / \mathrm{kg})$ immediately after the trans-septal puncture and continuous infusion of $2000 \mathrm{IU} / \mathrm{h}$ through a trans-septal sheath in order to obtain an activated clotting time (ACT) time result above $300 \mathrm{~s}$. ACT time was measured at 30-minute intervals during the procedure. Post-procedural 24-hour heparin infusion as per activated clotting time was continued only in patients with an international normalized ratio (INR) $<2$ during the procedure (lowest INR value: 1.8). Another vitamin $\mathrm{K}$ antagonist dose was administered $4 \mathrm{~h}$ after the procedure in order to obtain an INR within the range of 2.0-3.0 on the following day. Informed consent was obtained from each patient and the study protocol conforms to the ethical guidelines of the 1975 Declaration of Helsinki as reflected in a prior approval by the institution's human research committee. The project was accepted by the Bioethical Committee, Medical University of Silesia in Katowice (No. KNW/0022/KB1/72/13).

\section{Microembolic signal detection}

MES monitoring was carried out throughout the entire procedure: from the moment of large vessel puncture to the moment of device withdrawal. The right middle cerebral artery (MCA) was continuously insonated through a temporal bone window at a depth of $48-56 \mathrm{~mm}$ using a $2 \mathrm{MHz}$ probe, the Pioneer 2020. Embolic signals were evaluated by two independent observers in accordance with the established criteria [3, 19, 20]. Quantitative evaluations of MES were applied: grade I: 1-10 MES, grade II: $11-30$ and MES- curtain: > 30 signals [20]. An automated algorithm was used to differentiate between the gaseous and solid MES. The quantitative analysis was performed considering the procedure's division into four stages, as presented above.

\section{Magnetic resonance imaging (MRI) of the head}

The patients observed had their head MRIs performed twice: before and after ablation, with the maximum period between these tests being 12 months. The T2, FLAIR, SWI and DWI sequences were used to evaluate the lesions of vascular origin. The Fazekas scale (FS) was used to analyze the lesions in the white matter [21, 22]. The modified Fazekas scale ranges from mild to severe. Mild white matter hyperintensities $(\mathrm{WMH})$ (grade 1) was defined by punctate lesions in the deep white matter with a maximum diameter of $9 \mathrm{~mm}$ for a single lesion and of $20 \mathrm{~mm}$ for grouped lesions. Moderate WMH (grade 2) were early confluent lesions of $10-20 \mathrm{~mm}$ single lesions and $>20 \mathrm{~mm}$ grouped lesions in any diameter, and no more than connecting bridges between the individual lesions. Severe WMH (grade 3) were single lesions or confluent areas of hyperintensity of $\geq 20 \mathrm{~mm}$ in any diameter.

\section{Screening cognitive examination}

A neurology examination on each patient was carried out three times by neurologists: on the day of ablation and on the days of head MRIs. The Mini Mental State Examination Scale was performed twice, during the qualification and the last neurological examination [23].

\section{Statistical analysis}

For the description of parametric data, the mean, standard deviation, median, minimum and maximum value of a given variable in the study group were calculated. To characterize non-parametric data, the number of occurrences of the variable in the study group and the percentage of these occurrences were used.

\section{Results}

Eighty-eight patients were initially enrolled in the study; however, for technical reasons, the signal from MCA, which would have enabled us to analyze potential embolic signals, could not be obtained in 8 patients. Thus, the study enrolled 80 patients at a mean age of 58 years. The mean $\mathrm{CHA}_{2} \mathrm{DS}_{2}$ VASc score for the study group was 2 and indicated a low risk of embolic cardiovascular events in a 1-year follow-up. In the majority of patients, head MRIs did not reveal any vascular brain damage. The results of the screening cognitive function examination showed normal status in all patients. The demographic, clinical and radiological data of the patients are presented in Table I.

\section{Microembolic signals}

Microembolic signals during the monitoring of the flow in the right MCA were recorded in $61(76.25 \%)$ patients in the amount of $51-489$ (mean: 239). Most often these were registered during the $2^{\text {nd }}$ and $3^{\text {rd }}$ stage, most rarely during stage 1 . In the majority of patients (89\%), MES were gaseous in nature (Table II, Figure 1). The distribution of volume-MES analysis results was as follows: $1-10$ MES in 21 (33.43\%) patients, 11-30 in $34(55.73 \%)$ and MES- curtain (> 30 signals) in $6(10.84 \%)$ patients.

Evaluation of lesions in the white matter using the Fazekas scale at baseline and at 12 months of follow-up. The mean period between the $1^{\text {st }} \mathrm{MRI}$ and ablation: $2.8 \pm 1.15$ months; between ablation and the follow-up MRI: 10.4 months (9-11). The 
Table I. Demographic and clinical features of patients

\begin{tabular}{|c|c|}
\hline Parameter & Value \\
\hline Age, mean (range) & $58(50-63)$ \\
\hline Sex F/M, $n$ & $30 / 50$ \\
\hline \multicolumn{2}{|l|}{ AF duration [years], $n(\%)$ : } \\
\hline $0-5$ & $37(46)$ \\
\hline $6-10$ & $27(34)$ \\
\hline$>10$ & $16(20)$ \\
\hline Functional classification by EHRA & $3(2-3)$ \\
\hline $\mathrm{CHA}_{2} \mathrm{DS}_{2}$-Vasc risk scale, mean (range) & $2(0-4)$ \\
\hline CHD, $n(\%)$ & $15(19)$ \\
\hline $\mathrm{AH}, n(\%)$ & $56(70)$ \\
\hline $\mathrm{DM}, n(\%)$ & $15(19)$ \\
\hline $\mathrm{LD}, n(\%)$ & $55(69)$ \\
\hline Obesity, $n(\%)$ & $27(34)$ \\
\hline PAD, $n(\%)$ & $22(18)$ \\
\hline \multicolumn{2}{|l|}{ WMH, $n(\%):$} \\
\hline $0-1$ & 67 \\
\hline $2-3$ & 13 \\
\hline MMSE & $29(27-30)$ \\
\hline
\end{tabular}

F-female, M-male, AF-atrial fibrillation, EHRA - European Heart Rhythm Association, CHD - coronary heart disease, $A H$ - arterial hypertension, DM - diabetes mellitus, $L D$ - lipid disorders, PAD peripheral artery disease, $\mathrm{WMH}-$ white matter hyperintensities.

mean score on FS for the whole group before $a b-$ lation was: $0.87 \pm 0.7(0-3$, med. 1$)$; after ablation: $0.93 \pm 0.71$ (0-3, med. 1) (Figures 2, 3). Pre-ablation examination most often presented lesions that were not very advanced, scoring up to 1 point

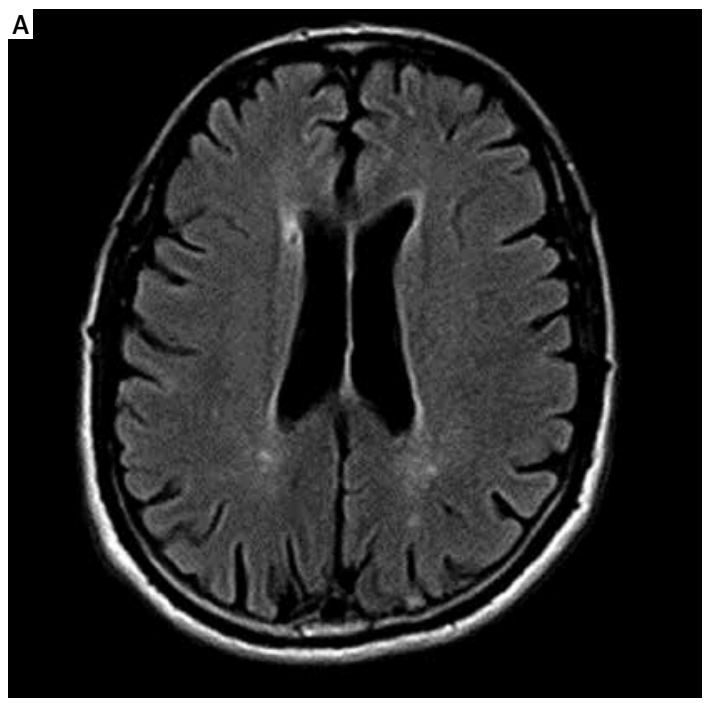

Figure 2. Vascular lesions in the Fazakas scale 1 grade
Table II. Characteristics of microembolic signals

\begin{tabular}{|lcc|}
\hline Parameter & $N$ (patients) & $N$ (MES) \\
\hline $\begin{array}{l}\text { MES, mean } \\
\text { (min.-max.); med. }\end{array}$ & 61 & $\begin{array}{c}263.6 \pm 117.9 \\
(51-489) ; 239\end{array}$ \\
\hline $\begin{array}{l}\text { Stage 1 } \\
\text { (min.-max.); med. }\end{array}$ & 14 & $\begin{array}{c}1.64 \pm 0.93 \\
(1-4) ; 1.0\end{array}$ \\
\hline \begin{tabular}{lcc|} 
Stage 2 \\
(min.-max.); med.
\end{tabular} & 61 & $\begin{array}{c}136.4 \pm 73.9 \\
(25-312) ; 111\end{array}$ \\
\hline Stage 3 & & $\begin{array}{c}102.8 \pm 69.8 \\
\text { (min.-max.); med. }\end{array}$ \\
\hline $\begin{array}{l}\text { Stage 4 } \\
\text { (min.-max.); med. }\end{array}$ & 50 & $\begin{array}{c}13.3 \pm 20.0 \\
(1-70) ; 5\end{array}$ \\
\hline $\begin{array}{l}\text { Gaseous } \\
\text { (min.-max.); med. }\end{array}$ & 26 & $\begin{array}{c}66.8 \pm 6.4 \\
(48-84) ; 93.1\end{array}$ \\
\hline
\end{tabular}

MES - microembolic signals.

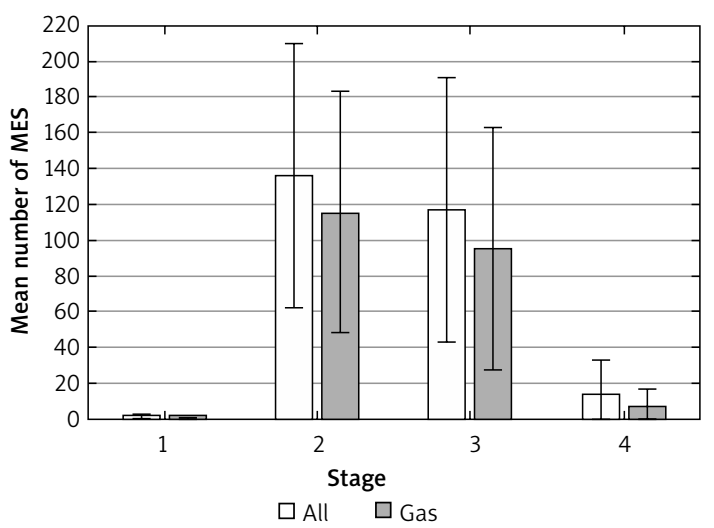

Figure 1. Distribution of microembolic signals (MES) over individual stages of the procedure

(71 patients, 95.7\%). In 3 (4.3\%) patients the lesions worsened (incrementing by 1 point in each patient) during the follow-up period. All lesions were asymptomatic. In 2 patients with worsening lesions of vascular origin, MES were recorded

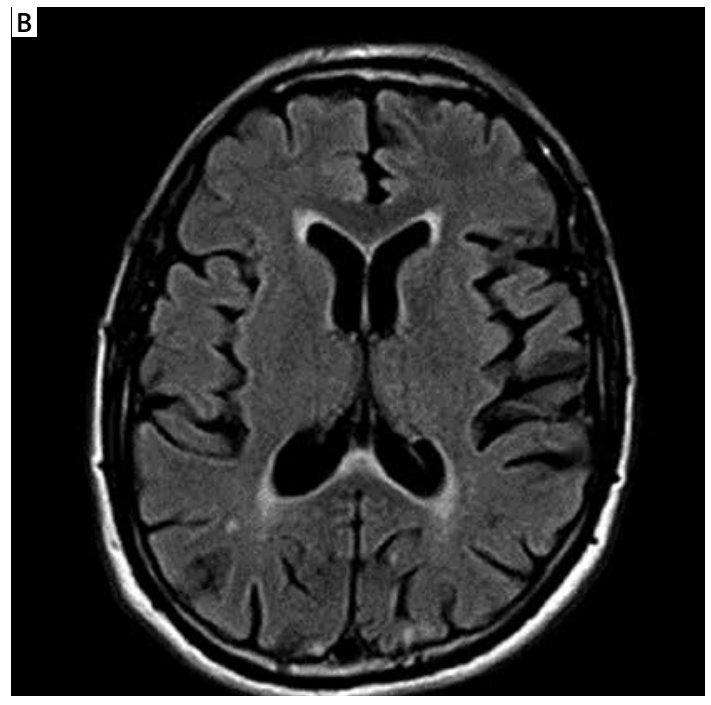




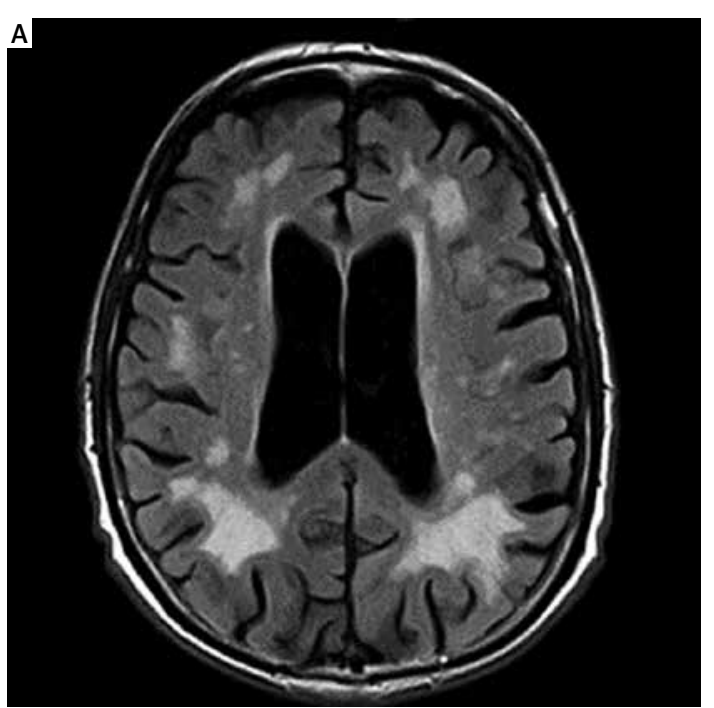

Figure 3. Vascular lesions in the Fazekas scale 3 stage

during ablation; these were gaseous and in the amount of 58 and 194.

\section{Evaluation of neurological status at baseline and at 12 months of follow-up}

During ablation, one patient reported a headache during stages 2 and 3; 1 patient reported a vision disorder that resolved within $<1$ min (septal puncture); the remaining patients did not report any neurological symptoms during the procedure.

None of the patients revealed a symptomatic cardiovascular event during the follow-up period and no changes were observed in patients' neurological statuses.

\section{Discussion}

The most important results of our study were as follows: MES are generated during PVI in the majority of patients. Most often, MES are gaseous in nature. Despite the occurrence of MES, PVI seems to be clinically safe. MES do not cause any chronic morphological damage to the nervous tissue visible in MRI of the head or any changes in the neurological and functional status of patients in the follow-up. The majority of MES appear during trans-septal puncture and directly after PVI. Based on the above information, the MES associated with PVI in most cases probably result from the technical aspects of the procedure and can occur during its various stages.

It has been shown in animal studies that during the introduction of a ring catheter the air forced into the left atrium through the septal sheath is the source of gaseous MES [7, 8, 16, 24]. Due to their unsteady structure and the ability to dissolve in the plasma, gaseous MES are rarely the cause of nerve tissue damage [16]. Although a significant amount of gaseous MES can be generated within

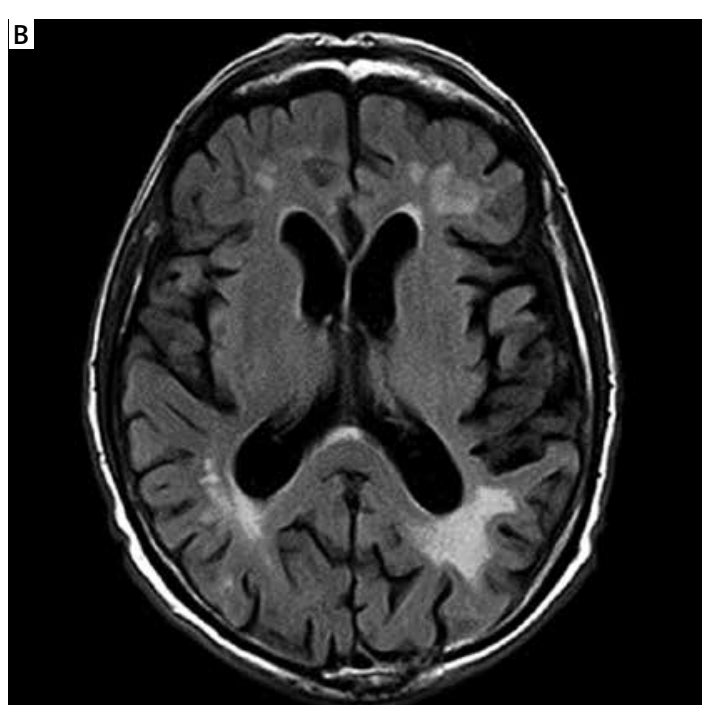

a short amount of time, their gaseous nature can probably explain the lack of symptoms. It seems, therefore, that most MES occur as a result of "iatrogenic gas injection". Another possible source of MES may be particulate debris (coagulum). Tissue overheating is also significant during the procedure. During ablation, German authors observed mainly equivocal and solid MES, these being also mostly asymptomatic [1]. Since those who qualified for ablation are patients free from thrombus, which is always verified in the TEE before PVI treatment, it should be ruled out that MES are formed with the embolic material generated from a "chronic" intracardiac thrombus. It is difficult to entirely exclude the presence of such material in other parts of the circulatory system in patients with AF. However, it should be emphasized that in our study population there were no patients with symptoms of heart failure or impaired left ventricular ejection fraction, which further limits the risk of a cardiogenic cause of embolism. On the other hand, it is worth considering that patients with AF are characterized by a state of endogenous hypercoagulability. The presence of micro-thrombi may result from coagulopathy. Potentially enhanced endogenous anticoagulant activity and the "consumption" of C and S proteins consequently lead to impaired anticoagulant protection [25]. Additionally, the activation of inflammatory processes may be of some importance. Our earliest observations indicated that the maneuvers in the left atrial immediately preceding PVI are associated with a pronounced inflammatory response, while the latter is exclusively related to impaired fibrinolysis [26].

To date, DWI has been used to evaluate cerebral lesions of vascular origin in patients after ablation in the peri-procedural period. It is worth emphasizing that this method facilitates visual- 
ization of ischemic foci within a few minutes of their onset. It is currently known that not every focus which occurs in DWI will evolve into a chronic ischemic focus. With DWI, ultra-acute lesions are visualized at the stage of cytotoxic edema caused by ischemia, and acute lesions are visualized after ischemic damage to the blood-brain barrier. According to the results of neuroimaging studies, acute lesions of vascular origin develop during ablation in $<20 \%$ of patients. Such lesions therefore serve as a radiological marker of MES. However, it has not been established whether they undergo further changes typical of stroke lesions. It was demonstrated that lesions $>10 \mathrm{~mm}$ occurring in the peri-procedural period are still present after $>12$ weeks, which may indicate that their effect on the nervous tissue lasts longer [6]. A comparative assessment of brain structures before and after ablation in the present study enabled us to analyze the potential evolution of the lesions of vascular origin and allowed us to assess the real burden associated with the procedure. Only 4\% of patients demonstrated progression of vascular lesions associated with short peri-procedural or later periods. It is difficult to precisely indicate the time of when the new lesions occur. Symptomatic and asymptomatic foci of damage to the nervous tissue appear in patients with AF. None of our patients experienced a clinical neurological event; only a repeated MRI revealed the presence of new chronic radiological lesions. For this study, we chose a rather homogeneous group of patients, with a low average risk of embolism during the follow-up period, in order to eliminate the causes of embolic events other than those potentially associated with ablation.

Although most authors on the subject have failed to show any correlation between the quality of lesions in MRI and the number of MES, there are some opposing data $[3,5,6]$.

Some authors suggest a cause-and-effect relationship between MES during ablation and cognitive disorders of varying severity. Verbal memory disorders were observed in the 3-month period following irrigated RF PVI [10]. However, these events were not confirmed in the MACPAF study [27]. The results of long-term follow-up are inconsistent in relation to the potential effect of MES during ablation on the intellectual deterioration [1, 3, 9, 28, 29]. Varying study protocols may have caused discrepancies in the results. Other parameters interfering with the results are also important; these include a concomitant intellectual dysfunction of a different etiology, older age and $A F$ with clinically silent strokes $[16,30]$. We enrolled patients with no cognitive or motor deficits in order to eliminate the influence of other potential parameters. We did not find any lesions in the above areas, which is consistent with the results of a radiological analysis related to our patients. The results of this study suggest that MES during ablation are associated with the technical aspects of the procedure and do not correspond to the burden of clinical embolism related to the procedure. MES monitoring does not allow us to determine the actual risk of cardiogenic cerebral embolism associated with ablation. The causes of cerebral ischemia in patients with cardiovascular diseases are multifactorial and differ among individual patients with atrial fibrillation. They are dependent on the clinical and pharmacological profile of the patient [31, 32]. The study's advantage was a standardized anticoagulant treatment using VKA with INR in therapeutic ranges. Patients were prepared for AF ablation by optimal anticoagulation according to the valid guidelines [33]. We did not study patients with non-vitamin $\mathrm{K}$ antagonist oral anticoagulants (NOAC), but in this case, it allowed us to objectify the results obtained [33]. The assessment of ablation safety in the aspect of potential damage to the nervous system during the procedure becomes more important when various methods of this procedure are available [34].

We are aware of some limitations of our study. The most important are as follows: unilateral detection of embolic signals (technical aspects of device), non-unified method for PVI (both RF ablation and balloon cryoablation), and a rather small group of patients without a control group. However, our research paper is the first reporting the results of a planned large clinical group. To evaluate the cognitive status we used a test of low sensitivity, but it is validated, well-known, and popular in clinical practice.

In conclusion, the majority of cerebral microembolism generated during PVI are gaseous in nature. The cerebral microembolisms associated with PVI probably result from the technical aspects of the procedure and do not cause either permanent brain damage in the radiological investigation or neurological deficit.

\section{Conflict of interest}

The authors declare no conflict of interest.

\section{References}

1. von Bary C, Deneke T, Arentz T, et al. Online measurement of microembolic signal burden by transcranial Doppler during catheter ablation for atrial fibrillation results of a multicenter trial. Front Neurol 2017; 8: 131.

2. Kuck KH, Brugada J, Fürnkranz A, et al.; FIRE AND ICE Investigators. Cryoballoon or radiofrequency ablation for paroxysmal atrial fibrillation. N Engl J Med 2016; 374: 2235-45.

3. Kochhäuser S, Lohmann HH, Ritter MA, et al. Neuropsychological impact of cerebral microemboli in ablation of atrial fibrillation. Clin Res Cardiol 2015; 104: 234-40. 
4. Cappato R, Calkins H, Chen SA, et al. Worldwide survey on the methods, efficacy, and safety of catheter ablation for human atrial fibrillation. Circulation 2005; 111: 1100-5.

5. Gaita F, Leclercq JF, Schumacher B, et al. Incidence of silent cerebral thromboembolic lesions after atrial fibrillation ablation may change according to technology used: comparison of irrigated radiofrequency, multipolar nonirrigated catheter and cryoballoon. J Cardiovasc Electrophysiol 2011; 22: 961-8.

6. Herrera Siklódy C, Deneke T, Hocini M, et al. Incidence of asymptomatic intracranial embolic events after pulmonary vein isolation: comparison of different atrial fibrillation ablation technologies in a multicenter study. J Am Coll Cardiol 2011; 58: 681-8.

7. Haines DE, Stewart MT, Dahlberg S, et al. Microembolism and catheter ablation I: a comparison of irrigated radiofrequency and multielectrode-phased radiofrequency catheter ablation of pulmonary vein ostia. Circ Arrhythm Electrophysiol 2013; 6: 16-22.

8. Kiss A, Nagy-Baló E, Sándorfi G, Edes I, Csanádi Z. Cerebral microembolization during atrial fibrillation ablation: comparison of different single-shot ablation techniques. Int J Cardiol 2014; 174: 276-81.

9. Newman SP, Harrison MJ. Coronary-artery bypass surgery and the brain: persisting concerns. Lancet Neurol 2002; 1: 119-25.

10. Schwarz N, Kuniss M, Nedelmann $M$, et al. Neuropsychological decline after catheter ablation of atrial fibrillation. Heart Rhythm 2010; 7: 1761-7.

11. Schrickel JW, Lickfett L, Lewalter T, et al. Incidence and predictors of silent cerebral embolism during pulmonary vein catheter ablation for atrial fibrillation. Europace 2010; 12: 52-7.

12. Wieczorek $M$, Lukat $M$, Hoeltgen $R$, et al. Investigation into causes of abnormal cerebral MRI findings following PVAC duty-cycled, phased RF ablation of atrial fibrillation. J Cardiovasc Electrophysiol 2013; 24: 121-8.

13. Nagy-Baló E, Tint D, Clemens M, et al. Transcranial measurement of cerebral microembolic signals during pulmonary vein isolation: a comparison of two ablation techniques. Circ Arrhythm Electrophysiol 2013; 6: 473-80.

14. Nagy-Balo E, Kiss A, Condie C, Stewart M, Edes I, Csanadi Z. Predictors of cerebral microembolization during phased radiofrequency ablation of atrial fibrillation: role of the ongoing rhythm and the site of energy delivery. Pacing Clin Electrophysiol 2014; 37: 1436-41.

15. Bittner A, Mönnig G, Zellerhoff S, et al. Randomized study comparing duty-cycled bipolar and unipolar radiofrequency with point-by-point ablation in pulmonary vein isolation. Heart Rhythm 2011; 8: 1383-90.

16. Miyazaki S, Watanabe T, Kajiyama T, et al. Thromboembolic risks of the procedural process in second-generation cryoballoon ablation procedures: analysis from real-time transcranial doppler monitoring. Circ Arrhythm Electrophysiol 2017; 10: pii: e005612.

17. Wieczorek $M$, Hoeltgen R, Brueck $M$. Does the number of simultaneously activated electrodes during phased RF multielectrode ablation of atrial fibrillation influence the incidence of silent cerebral microembolism? Heart Rhythm 2013; 10: 953-9.

18. Demir GG, Güneş HM, Seker M, et al. Is the presence of left atrial diverticulum associated with recurrence in patients undergoing catheter ablation for atrial fibrillation? Arch Med Sci Atheroscler Dis 2019; 4: e25-31.

19. Ringelstein EB, Droste DW, Babikian VL, et al. Consensus Committee of the Ninth International Cerebral Hemody- namic Symposium. Basic identification criteria of Doppler microembolic signals. Stroke 1995; 26: 1123.

20. Consensus on microembolus detection by TCD. International Consensus Group on Microembolus Detection. Stroke 1998; 29: 725-9.

21. Lao AY, Sharma VK, Tsivgoulis G, et al. Detection of rightto-left shunts: comparison between the International Consensus and Spencer Logarithmic Scale criteria. J Neuroimaging 2008; 18: 402-6.

22. Pantoni L, Basile AM, Pracucci G, et al. Impact of age-related cerebral white matter changes on the transition to disability: the LADIS study: rationale, design and methodology. Neuroepidemiology 2005; 24: 51-62.

23. Norris D, Clark MS, Shipley S. The mental status examination. Am Fam Physician 2016; 94: 635-41.

24. Takami M, Lehmann HI, Parker KD, Welker KM, Johnson SB, Packer DL. Effect of left atrial ablation process and strategy on microemboli formation during irrigated radiofrequency catheter ablation in an in vivo model. Circ Arrhythm Electrophysiol 2016; 9: e003226.

25. Negreva M, Georgiev S, Vitlianova K. Decreased activity of the protein $C$ anticoagulant pathway in the early hours of paroxysmal atrial fibrillation. Clin Appl Thromb Hemost 2017; 23: 793-9.

26. Wybraniec MT, Wieczorek J, Woźniak-Skowerska I, et al. Atrial fibrillation and rapid atrial pacing are linked to enhanced inflammation and altered fibrinolysis in patients undergoing pulmonary vein isolation. Kardiol Pol 2019; 77: 386-8.

27. Herm J, Fiebach JB, Koch L, et al. Neuropsychological effects of MRI-detected brain lesions after left atrial catheter ablation for atrial fibrillation: long-term results of the MACPAF study. Circ Arrhythm Electrophysiol 2013; 6: 843-50.

28. Browndyke JN, Moser DJ, Cohen RA, et al. Acute neuropsychological functioning following cardiosurgical interventions associated with the production of intraoperative cerebral microemboli. Clin Neuropsychol 2002; 16: 463-71.

29. Medi C, Evered L, Silbert B, et al. Subtle post-procedural cognitive dysfunction after atrial fibrillation ablation. J Am Coll Cardiol 2013; 62: 531-9.

30. Wieczorek J, Mizia-Stec K, Lasek-Bal A, et al. CHA2DS2Vasc score, age and body mass index as the main risk factors of hyperintense brain lesions in asymptomatic patients with paroxysmal non-valvular atrial fibrillation. Int J Cardiol 2016; 215: 476-81.

31. Kotlęga D, Gołąb-Janowska M, Meller A, et al. Beneficial effects of pre-stroke statins use in cardioembolic stroke patients with atrial fibrillation: a hospital-based retrospective analysis. Arch Med Sci 2019; 15: 385-92.

32. Ying C, Wang S, Lu Y, et al. Glucose fluctuation increased mesangial cell apoptosis related to AKT signal pathway. Arch Med Sci 2019; 15: 730-7.

33. Calkins H, Hindricks G, Cappato R, et al. 2017 HRS/EHRA/ ECAS/APHRS/SOLAECE expert consensus statement on catheter and surgical ablation of atrial fibrillation. Europace 2018; 20: 1-160.

34. Koźluk E, Piątkowska A, Rodkiewicz D, Peller M, Ko chanowski J, Opolski G. Direct results of a prospective randomized study comparing ablation with the $\mathrm{nMARQ}$ catheter and the PVAC catheter used with and without a 3D system (MAPER 3D Study). Arch Med Sci 2019; 15: 78-85. 